\title{
DISASTER RESILIENCE OF PLACE (DROP) MODEL
}

\section{A Resilience Assessment and Improvement Framework (RAIF) for facilities managers}

\author{
Keith G. Jones*
}

\section{Background}

Disaster events, whether natural (flood, heatwave, earthquakes, pandemics etc.) or human-made (cyber-attack, terrorism etc.), have a significant impact on the performance of business organisations (private, public and not-for-profit) and on the health and well-being of the communities to which they belong. How organisations prepare for, respond to and recover from these impacts depends on their vulnerability and resilience to the disaster event. Those organisations that exhibit a low vulnerability and high resilience tend to recover quickly, using the experiences they gain to inform their preparedness and identify mitigation actions to reduce their vulnerability and/or enhance their resilience to a future event. Those organisations that exhibit a high vulnerability and low resilience tend to recover more slowly, or in many cases do not recover at all.

\subsection{Resilience}

The concept of resilience was introduced by Holling $(1973,1996,2001)$ to describe the fluctuations in ecological systems exposed to external disturbance (disaster event) over time. Holling $(1973,1996)$ argued that such systems demonstrate two aspects of resilience: engineering resilience and ecological (later socio-ecological) resilience. Engineering resilience describes the system's behaviour close to its pre-existing equilibrium point, using resistance to the disturbance and speed of return to the equilibrium point as measures of the system's resilience. In essence, engineering resilience is concerned with retaining the stability of the system during and after a disaster event. Ecological resilience describes the reorganisation potential of the system to a new state of equilibrium following an external disturbance and is concerned with the magnitude of the disturbance that the system can absorb before reorganisation occurs. In essence, ecological resilience is concerned with flexibility/rigidity of the system to a disaster event. Holling (2001) extended the concept of ecological resilience to social-ecological systems, identifying the role that resilience and vulnerability play in triggering creative (desirable) reorganisation (change) or resisting destabilising (undesirable) change. In essence, social-ecological resilience is concerned

^keith.jones@aru.ac.uk 
with the inherent potential (adaptive capacity) of the system to change as a result of a disaster event. Since 2001 the various resilience concepts have been applied to help understand urban resilience at different spatial and temporal scales, population, environment, organisational, physical, lifestyle, economic and socio-cultural; (Cimellaro, 2016) as well as to the systems (infrastructure, lifelines, business, etc.) that modern society relies on for their existence and prosperity (Paton \& Johnston, 2017). In this chapter, the focus will be on business resilience.

\subsection{Vulnerability}

Whilst resilience is primarily concerned with the ability of a system to resist, absorb, accommodate, adapt to, transform and recover from a disaster event; vulnerability is concerned with the susceptibility of a system to a specific disaster threat (Bakkensen et al., 2016). The UNDRR (2020) define vulnerability as "The conditions determined by physical, social, economic and environmental factors or processes which increase the susceptibility of an individual, a community, assets or systems to the impacts of hazards". However, as vulnerability, hazard and exposure are the fundamental components of disaster risk (Preventionweb, 2020), vulnerability can also be viewed as the potential losses that a system would experience as a consequence of a disaster event (Murnane et al., 2016) which is related to resilience through a focus on disaster risk reduction (Zhou et al., 2016). In essence, the more resilient a system is, the less vulnerable it is to the impacts of a disaster event.

\subsection{Disaster Resilience of Place (DROP) model}

Cutter et al. (2008) explored the relationship between vulnerability and resilience to disaster events in her Disaster Resilience of Place (DROP) model (Figure 6.1).

The DROP model views vulnerability and resilience as separate but linked concepts, arguing that the vulnerability/resilience of a system to a disaster event is defined by the antecedent relationships (inherent vulnerability and resilience) that exist between the interaction of natural systems, social systems and the built environment (a system of systems model). The antecedent conditions interact with the disaster event characteristics (which will vary depending on the nature of the disaster event, geographical location, etc.) and the immediate effects of the disaster

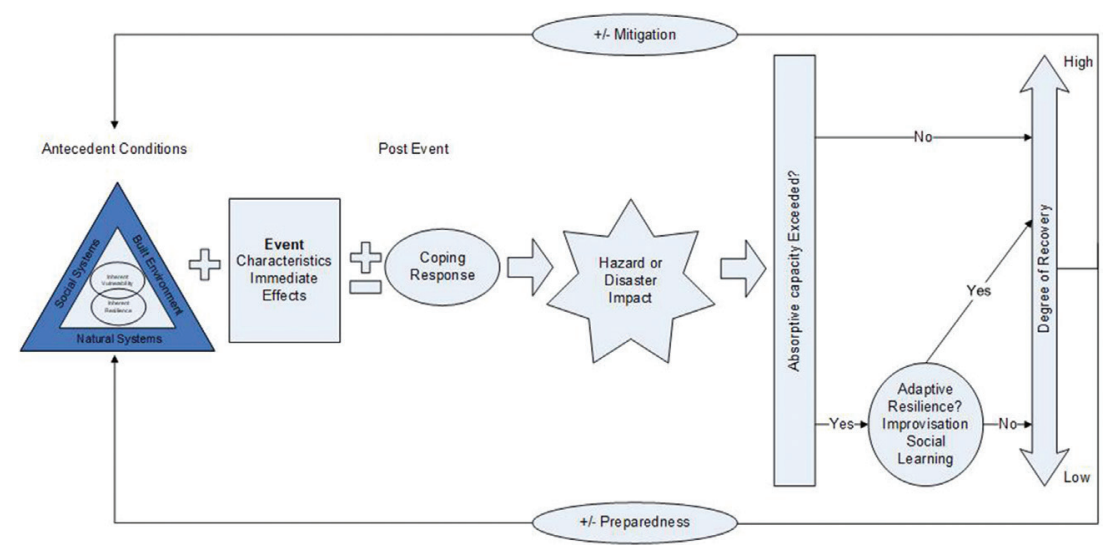

Figure 6.1 The DROP model

Source: Cutter et al. (2008) 
event are realised. These effects can be reduced (or amplified) by any post-event coping measures (e.g. disaster management and resilience plans), and the full disaster impact is realised. The impact of the disaster event on a system is moderated by the absorptive capacity of the system. If the absorptive capacity is not exceeded, then recovery is relatively quick. If the absorptive capacity is exceeded, then the system either adapts, and recovery occurs relatively quickly, or doesn't adapt, and recovery is slower or in extreme cases does not occur. Finally, if mitigation and preparedness occur, then the antecedent conditions are improved ahead of the next occurrence of the disaster event.

Cutter et al. (2010) applied the DROP model to assess the resilience of communities in the south-eastern United States, developing a range of metrics to measure social, economic, institutional, infrastructure and community competence resilience factors that allowed her to identify patterns of resilience and identify mitigation strategies to improve disaster resilience. Cutter et al. (2010) confirmed the ability of the DROP model to establish a baseline measurement of the characteristics that underpin community resilience and in turn to monitor changes in resilience over time in a particular place and to compare the disaster resilience of one place to another. Jones et al. (2013) applied the DROP model to examine the impacts of climate change on community resilience to extreme weather events in South-east London (Jones \& Ali, 2013). Jones et al. (2013) integrated the concepts underpinning the DROP model within a risk assessment framework to identify the vulnerability and resilience of a public housing organisation to extreme weather events (flooding and overheating). Through this work, Jones et al. (2017) demonstrated that although the DROP model was originally developed for a community scale, it could be applied at an organisational scale to identify the vulnerability and improve the resilience of a public housing organisation through the identification and integration of building adaptations into the built asset management process (Jones et al., 2017). In the LIQUEFACT project, Morga et al. (2020) extended the application of the DROP model to a wider range of organisation types, developing the Resilience Assessment and Improvement Framework (RAIF) as a conceptual model which researchers could use to develop the tools that business organisations need to assess their vulnerability, and improve their resilience, to earthquake-induced liquefaction events. ${ }^{1}$

\section{Application to workplaces}

Organisational resilience "is the ability of an organisation to anticipate, prepare for, respond and adapt to, incremental change and sudden disruptions in order to survive and prosper" (Denyer, 2017). Resilient organisations demonstrate the ability to defend their organisation through preventative control and mindful action and take advantage of disruptive opportunities through performance optimisation and adaptive innovation (Denyer, 2017). This said, understanding the impact that a disaster event has on organisational resilience is an under-researched area that has a major impact on both organisational survival (Gibson \& Tarrant, 2010) and community resilience (UNDRR, 2015). The RAIF was specifically developed to provide a decision framework to help business organisations better understand their vulnerabilities and resilience to disaster events.

\subsection{Understanding organisational resilience}

The author would argue that business organisations, in both public and private sectors, exhibit many of the characteristics of complex adaptive systems. At the physical level, business organisations rely on physical assets (e.g. buildings, information communication technology [ICT], etc.) for the production and delivery of their primary services (Hard FM systems); whilst at the operational level, they rely on socio-economic assets (e.g. human, financial, etc.) to support 
their primary services (Soft FM systems). However, business organisations also form part of the wider community ecosystem, relying for their survival on other organisations within their supply chain and on providing employment, goods and services to the wider community (business and FM strategy). Further, given that business organisations exist as part of a system of systems, to understand their inherent resilience to disaster events, and to identify mitigation actions that they can take to increase their resilience, they need to understand not only the impact that a disaster event could have on the functional performance of their organisation, but also on their organisation's relationship with its customers and the wider community. As such, business organisations (and particularly their facilities manager) need to adopt a hybrid view of resilience, where engineering resilience drives the functional performance of the organisation's physical assets, and social-ecological resilience drives the functional performance of its services and wider business and community relationships.

\subsection{Theory underpinning the RAIF}

The RAIF provides a set of tools to help facilities managers examine what mitigation and preparedness actions should be taken to reduce vulnerability and improve resilience to a disaster event; and guidance on how to integrate these actions into facilities management (FM) strategies and plans. The RAIF integrates the DROP model (Cutter et al., 2008) with the strategic built asset management model (Figure 6.2) developed by Jones and Sharp (2007).

Strategic built asset management is the process by which organisations align their built assets with the strategic needs of their organisation through maintenance and refurbishment. Strategic built asset management uses a range of key performance indicators to routinely assess the ability of the organisation's built assets to support its primary business function expressed through critical success factors. Performance toolkits measure current performance and identify any obsolescence (the difference between current performance and desired performance from a business perspective), and analysis toolkits establish the cause of any obsolescence and identify improvements in performance needed to close the obsolescence gap (Action Statement). Modelling toolkits identify potential solutions (physical, operational, organisational) against the Action Statement brief, and those that are deemed cost effective (through an Options Appraisal process) are integrated into the organisation's strategic built asset management plans (maintenance, refurbishment and estate management plans). The RAIF (Figure 6.3) integrates disaster management and business resilience planning into the strategic built asset management process.

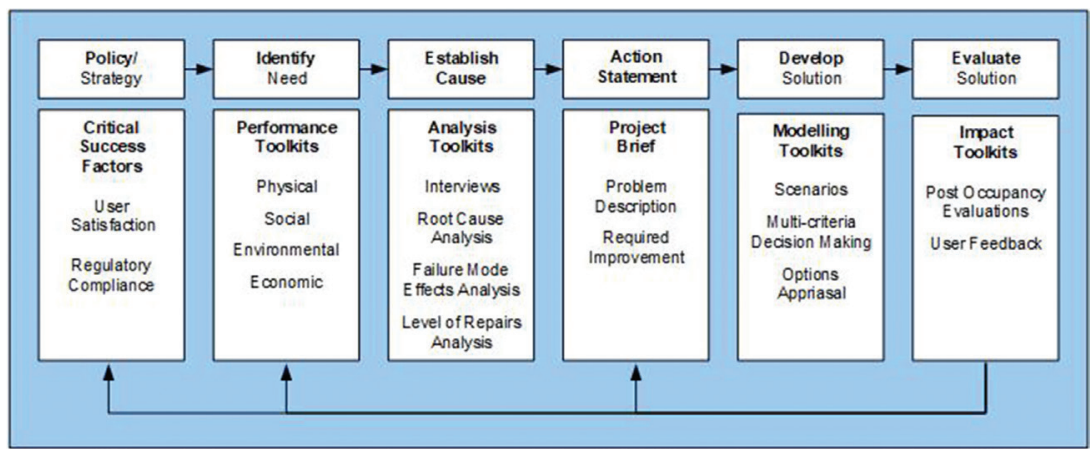

Figure 6.2 Performance-based built asset management Source: Jones and Sharp (2007) 


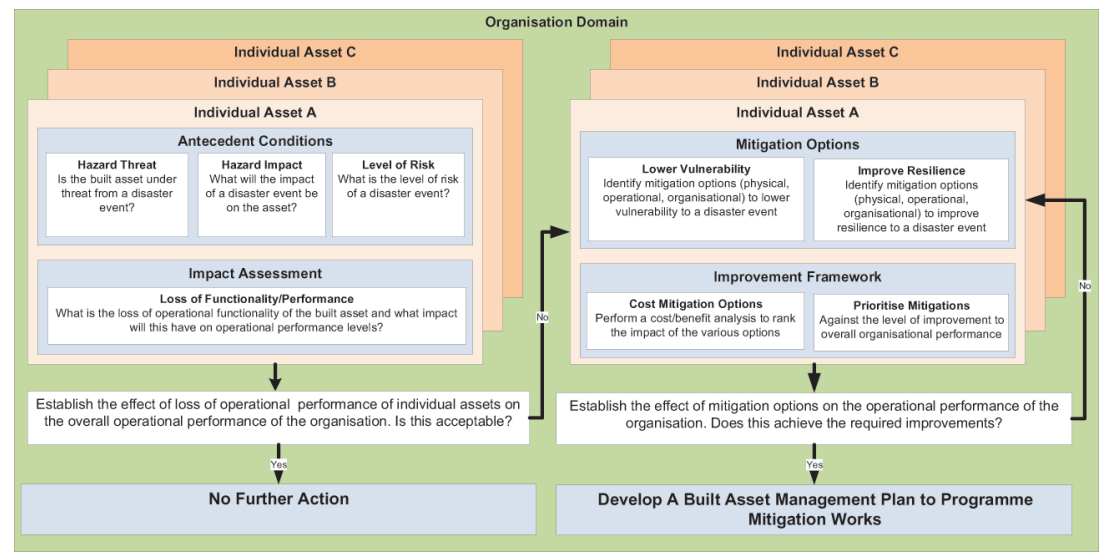

Figure 6.3 The Resilience Assessment and Improvement Framework

Source: Adapted from Morga et al. (2020)

The RAIF uses a range of performance toolkits (e.g. hazard assessments, resilience scorecards, etc.) to provide a series of key performance indicators against which the impact of different disaster scenarios (deterministic analyses) and risk models (probabilistic analyses) can be assessed. This provides the facilities manager with an assessment of the antecedent vulnerability and resilience of their organisation to a given disaster event.

Once the characteristics of the disaster event have been modelled, their effect on the functional performance of the organisation can be assessed by examining the impact that a disaster event characteristics would have on the physical (Hard FM) and operational (Soft FM) performance of the organisation. If the overall impact of the disaster event is acceptable to the organisation, then no further action is needed. If the overall impact is unacceptable (as measured against the organisation's critical success factors) then mitigation interventions (physical, operational, organisational) are needed to reduce vulnerability and/or improve resilience of the organisation to the disaster event. This step provides the facilities manager with a problem statement against which potential mitigation solutions can be evaluated.

The potential improvement in performance of different mitigation solutions can be assessed against different scenarios using multi-criteria analysis tools to assess their overall functional impact against the organisational critical success factors (individual mitigation interventions will impact a range of performance metrics). This step provides the facilities manager with a short list of potential mitigation interventions that can be explored in detail through an options appraisal process where cost-benefit analyses can identify those mitigation interventions that should be implemented as part of a disaster risk reduction improvement framework. This step provides the facilities manager with the business cases to evaluate the potential improvement in functional performance of the disaster mitigation interventions and supports the integration of the most effective (based on a cost/risk reduction basis) mitigations into the organisation's strategic built asset management plans.

\subsection{Impact on community resilience}

Business organisations do not exist in isolation but form part of a complex network of stakeholders (e.g. citizens, businesses, policymakers, legislators, etc.) whose collective response to a disaster event contributes significantly to a community's overall response to the disaster event. Immediately 
following a disaster event, communities rely very heavily on their built assets and infrastructure systems (e.g. healthcare, power supply, transport, water supply, education, commercial, retail, etc.) to support their recovery. The UN (United Nations) Sendai Framework for Disaster Risk Reduction (UNDRR, 2015) recognises the role that these multiple stakeholders, including business organisations, play in improving community resilience to a disaster event; identifying the need for all stakeholders to better understand the risks they face and developing a range of physical and operational mitigation interventions to reduce/manage these risks through a planned and coordinated response. In particular, the Sendai Framework identifies the need for organisations to:

- understand their disaster risk in all its dimensions and integrate this knowledge into risk assessment and disaster management/resilience plans,

- $\quad$ strengthen disaster risk governance to manage disaster risk including collaboration and partnerships,

- invest in disaster risk reduction for resilience through structural and non-structural measures, and

- enhance disaster preparedness and response to disaster events, including to "build back better" in recovery, rehabilitation and reconstruction.

The integration of the RAIF into risk management (see the vignette in Section 5) provides a step-by-step decision support framework for facilities managers to better understand their organisation's vulnerability and resilience to disaster risk and provides the business tools to identify business mitigation actions (both Hard and Soft FM interventions) to better manage the disaster risk and support both pre-disaster preparations and post disaster response. In this way, business organisations can make a positive contribution to overall community resilience at both the operational and strategic levels.

\section{Methodology/research approach}

The RAIF was developed by combining the DROP model (Cutter et al., 2008) with the performance-based built asset management model (Jones \& Sharp, 2007) using a mixed-method research methodology to develop a conceptual model of organisational resilience to earthquake-induced liquefaction and identify mitigation interventions that could be programmed into long-term built asset management plans. The conceptual model was developed through a series of interactive workshops with expert stakeholder groups representing both end users and researchers. The conceptual model was operationalised by six research teams who developed specific tools for each stage of the RAIF. The RAIF and its associated tools were validated against a series of hypothetical organisational scenarios, with both the conceptual model and tools being modified to reflect stakeholder feedback. Full details of the development process and associated tools can be found on the LIQUEFACT website (www.liquefact.eu).

Although the RAIF was developed against a specific hazard, it is generic in nature and could be applied by any organisation (or research project) to assess the impact that a natural hazard could have on organisational resilience. Indeed, the RAIF as described in this chapter is an extension of an earlier risk assessment framework developed to understand the impact that extreme weather events could have on the resilience of built assets (Jones et al., 2017). Further details of how to apply the RAIF to different hazard scenarios are given later in this chapter.

The RAIF does not address the socio-economic aspects of organisational resilience (e.g. size, financial health, market disruption, economic conditions) or wider community resilience implications. This latter point in particular is a clear gap in existing knowledge that needs to be 
addressed by considering organisational resilience as part of a wider corporate social responsibility agenda. The integration of the RAIF into a holistic community resilience framework is an area of ongoing research that the author has started to address by considering its potential integration into the UNDRR disaster resilience scorecard for cities (UNDRR, 2017) in the LIQUEFACT and TURNkey ${ }^{2}$ projects.

\section{Limitations}

Whilst the RAIF provides a conceptual model of organisational resilience to disaster events, the tools required to operationalise the model need to be developed to reflect the specific circumstances (hazard events, impact on functionality, mitigation options and attitudes to risk) that different organisations face. The time taken to translate the conceptual model into an operational model should not be underestimated. The development of the RAIF, in particular its operational tools, took many person-months, and whilst for some hazards the operational tools may already exist, for many others they do not. As such, it is probably beyond the scope of all but the largest organisations to undertake the full application of the RAIF; and it is more likely that small organisations would use the RAIF as a decision support framework to help them better understand the disaster risks that they face.

From a research perspective, it is also important to understand the complex interactions between the factors that affect an organisation's vulnerability, resilience and adaptive capacity. The RAIF adopts a built asset-centric perspective on disaster resilience to provide a framework through which facilities managers can better understand the impact that a natural disaster event has on service delivery.

Finally, whilst the RAIF provides a generic decision support framework that facilities managers can customise and apply to any disaster event, scenario facilities managers should not underestimate the time and cost resources required by an organisation to customise the RAIF for their particular context and disaster event scenario.

\section{Theory relevance for practice}

The RAIF was developed over two research projects to provide a practical decision support framework that facilities managers can customise against different disaster scenarios to support the development of effective built asset management, disaster management and business continuity and resilience plans. The RAIF was operationalised and validated against two disaster scenarios; earthquake induced liquefaction and extreme weather events. Through both of these scenarios, the RAIF was presented as a 10-step Facilities Management Disaster Risk Reduction framework (Figure 6.4)

The RAIF was applied to assess the resilience of a hypothetical hospital to an earthquakeinduced liquefaction disaster event (EILD) as part of the EU H2020 LIQUEFACT project. Earthquake-induced liquefaction occurs when saturated granular soils lose the majority of their strength as a consequence of ground shaking, causing buildings to sink and/or overturn. The following vignette presents a simplified version of the scenario developed in the LIQUEFACT project.

Organisations are complex adaptive systems, and as such, assessing their resilience to a disaster event and for facilities managers to evaluate their organisations exposure to disaster events requires them to understand the vulnerability, resilience, and adaptive capacity of their organisation to a disaster event. In developing this understanding, facilities managers need to consider not only the impact of a disaster event on their organisation's Hard and Soft FM systems but also on their overall business continuity during and after a disaster event. To support this understanding, facilities managers need to adopt a social-ecological view of resilience where engineering resilience (stability) is combined with ecological resilience (flexibility/rigidity) to reflect the ability of 


\begin{tabular}{ll}
\hline Step & Activity \\
\hline 1 & Set the strategic goals and operational objectives for the plan \\
2 & Identify the built assets that are susceptibility to a specific disaster event \\
3 & Assess level of risk for each susceptible built asset \\
4 & Estimate the damage profile of each susceptible built asset \\
5 & Estimate loss of functionality / performance of each built asset \\
6 & Estimate the effect of loss of performance on organisation and community resilience \\
7 & Identify technical (Hard FM) and operational (Soft FM) mitigation options \\
8 & Perform a Cost Benefit Analysis to shortlist a range of possible interventions \\
9 & Perform an Options Appraisal to prioritise the shortlisted mitigations \\
10 & Develop an implementation plan to programme mitigation work over the built asset life cycle \\
\hline
\end{tabular}

Figure 6.4 Generic 10 Facilities Management Step Disaster Risk Reduction framework

\section{Hypothetical Primary Health Care Scenario}

The facilities manager for a regional hospital was asked to assess the potential impact of an EILD event on the functioning of the hospital. The hospital is located on 2 sites across a small city. Each site contains a number of buildings that provide primary care, administrative and support services to the city community.

\section{Antecedent Conditions}

The facilities manager geolocates the hospital's built assets onto a European Liquefaction Hazard Map to identify those assets that are potentially exposed to EILD event. For each asset that is potentially exposed the facilities manager assesses the level of exposure against a medium (most probable) and high (most severe) earthquake scenario (characteristics). One of the hospital's sites is located in an earthquake zone where the generic ground conditions are prone to liquefaction. For the most probable scenario the hazard impact is low, and the level of risk is low. For the most severe scenario the hazard impact is high, and the level of risk is medium. This site was investigated further.

\section{Impact Assessment}

For the most probable scenario discussions between the facilities manager, building users and the health authorities technical consultants identify the likelihood of "minor cosmetic damage" to the buildings. For the most severe scenario discussions identify the likelihood of "moderate to severe structural damage" to the different buildings.

\section{Functionality}

For the most probable scenario the loss of functionality will be minor resulting in minimal impact on the performance of the hospital immediately following an EILD event. The hospital could be back to full performance levels once emergency clean-up operations were complete. As such the overall impact on the community will also be minor and no further investigation of this scenario is required. For the most severe scenario the loss of functionality will be major, requiring major repairs over a prolonged time resulting in the hospital services having to be diverted to other health care sector providers. Depending upon the overall impact of the earthquake on the healthcare sector (it is likely that the regional sector will have a healthcare resilience plan) the loss of functionality could have a major impact on community resilience. As such the facilities manager was been asked to identify mitigation options to reduce the risks associated with this scenario.

\section{Figure 6.5 Healthcare sector vignette}

Source: Adapted from Morga et al. (2020) 


\section{Mitigation Options}

Technical mitigation to the soil were identified to reduce the risk from medium to very low for all but one of the buildings, where the risk remained medium. Operational mitigation options were identified to relocate critical healthcare services from the medium risk building to one of the very-low risk buildings, using the medium risk building for storage. Organisational mitigation options were identified as part of medium term built asset management plan to demolish the medium risk building over the next 5-10 years and replace it with a very-low risk building. Organisational mitigation options were also identified to update the hospital's risk assessments, disaster management plan (DMP), and business continuity plans (BCP) to explicitly reflect an EILD event and ensure that the hospital identified the adaptive capacity (human, economic, management) to manage any disruption to service delivery and ensure that the hospital could absorb the impacts of an EILD event and recover quickly.

\section{Improvement Framework}

Following detailed cost/benefit analyses the most cost-effective technical mitigations were programmed into a 5-year built asset management plan and detailed design solutions were commissioned. Operational mitigations to relocate services and update the risk assessment, DMP and BRPs were instigated. All the mitigations were evaluated against the required functional improvements.

Figure 6.5 Continued

the organisation to re-organise (adaptive capacity) following a disaster event. The RAIF provides the conceptual basis to help facility management researchers (and practitioners) in this task.

\section{Acknowledgement}

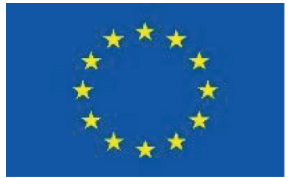

The LIQUEFACT project has received funding from the European Union's Horizon 2020 research and innovation programme under grant agreement No 700748.

\footnotetext{
This project has received funding from the European Union's Horizon 2020 research and innovation programme under grant agreement No. 700748
}

\section{Notes}

1 Earthquake-induced liquefaction occurs when soil strength and stiffness decrease as a result of increased pore water pressure in saturated granular materials during seismic ground motion, causing the soil to behave like a liquid and resulting in buildings and lifelines sinking, settling or overturning.

2 https://earthquake-turnkey.eu/

\section{Further reading}

Cimellaro, G. P. (2016). Urban Resilience for Emergency Response and Recovery: Fundamental Concepts and Applications. Switzerland: Springer.

Denyer, D. (2017). Organisational Resilience: A Summary of Academic Evidence, Business Insights and New Thinking. Cranfield, UK: BSI and Cranfield School of Management.

Jones, K. G. (2012). Preparing for extreme weather events: A risk assessment Approach. In C. Booth, F. Hammond, J. Lamond \& D. Proverbs (Eds.), Solutions to Climate Change Challenges in the Built Environment. West Sussex, UK: Wiley-Blackwell.

Paton, D., \& Johnston, D. (2017). Disaster Resilience: An Integrated Approach (2nd ed.). Springfield, IL: Charles C Thomas Publisher. 


\section{References}

Bakkensen, L. A., Fox-Lent, C., Read, L. K., \& Linkov, I. (2016). Validating resilience and vulnerability indices in the context of natural disasters. Risk Analysis, 37(5), 982-1004. doi:10.1111/risa.12677

Cimellaro, G. P. (2016). Urban Resilience for Emergency Response and Recovery: Fundamental Concepts and Applications. Switzerland: Springer.

Cutter, S. L., Barnes, L., Berry, M., Burton, C., Evans, E., Tate, E., \& Webb, J. (2008). A place-based model for understanding community resilience to natural disasters. Global Environmental Change, 18(4), 598-606. doi:10.1016/j.gloenvcha.2008.07.013

Cutter, S. L., Burton, G. B., \& Emrich, C. T. (2010). Disaster resilience indicators for benchmarking baseline conditions. Journal of Homeland Security and Emergency Management, 7(1). Article 51. doi:10.2202/1547-7355.1732

Denyer, D. (2017). Organisational Resilience: A Summary of Academic Evidence, Business Insights and New Thinking. BSI and Cranfield School of Management. www.cranfield.ac.uk/som/case-studies/ organizational-resilience-a-summary-of-academic-evidence-business-insights-and-new-thinking

Gibson, C. A., \& Tarrant, M. (2010). A 'conceptual models' approach to organisational resilience. Australian Journal of Emergency Management, 25(2). https://ajem.infoservices.com.au/items/AJEM-25-02-03

Holling, C. S. (1973). Resilience and stability of ecological systems. Annual Review of Ecology and Systematics, 4(1), 1-23. doi:10.1146/annurev.es.04.110173.00024

Holling, C. S. (1996). Engineering resilience versus ecological resilience, engineering within ecological constraints. National Academies of Sciences, Engineering, and Medicine, 31-44. www.nap.edu/read/4919/ chapter/4

Holling, C. S. (2001). Understanding the complexity of economic, ecological, and social systems, Ecosystems, 4, 390-405. doi:10.1007/s10021-001-0101-5

Jones, K., Desai, A., Brosnan, N., Cooper, J., \& Ali, F. (2017). Built asset management climate change adaptation model. International Journal of Disaster Resilience in the Built Environment, 8(3), 263-274. doi:10.1108/IJDRBE-07-2016-0032

Jones, K., \& Sharp, M. (2007). A new performance-based process model for built asset maintenance. Facilities, 25(13/14), 525-535. doi:10.1108/02632770710822616

Jones, K. G., \& Ali, F. (2013). Community resilience to extreme weather events through improved local decision making. In S. Hallett (Ed.), Community Resilience to Extreme Weather - the CREW Project Final Report (pp. 27-38). https://arcc.ouce.ox.ac.uk/wp-content/pdfs/CREW_Final_Report.pdf

Jones, K. G., Brydson, H., Ali, F., \& Cooper, J. (2013). Assessing vulnerability, resilience and adaptive capacity of a UK Social Landlord. International Journal of Disaster Resilience in the Built Environment, 4(3), 287-296. doi:10.1108/IJDRBE-03-2013-0004

Morga, M., Pascale, F., Wanigarathna, N., Majeed, Z., Meslem, A., \& Jones, K. (2020). Whole life built asset management modelling framework: Integrating mitigation to earthquake induced liquefaction disaster events into strategic built asset management. LIQUEFACT Deliverable 5.4. www.liquefact.eu/ wp-content/uploads/2018/12/D1.4-Detailed-user-requirements-and-research-output-protocols-forthe-LIQUEFACT-Reference-Guide.pdf

Murnane, R., Simpson, A., \& Jongman, B. (2016). Understanding risk: What makes a risk assessment successful. International Journal of Disaster Resilience in the Built Environment, 7(2), 186-200. doi:10.1108/ IJDRBE-06-2015-0033

Paton, D., \& Johnston, D. (2017). Disaster Resilience: An Integrated Approach (2nd ed.). Springfield, IL: Charles C Thomas Publisher.

Preventionweb. (2020). Disaster Risk. www.preventionweb.net/disaster-risk/risk/disaster-risk/

UNDRR. (2015). The Sendai Framework for Disaster Risk Reduction 2015-2030. www.undrr.org/ implementing-sendai-framework/what-sendai-framework

UNDRR. (2017). Disaster Resilience Scorecard for Cities. www.undrr.org/publication/disaster-resiliencescorecard-cities

UNDRR. (2020). Vulnerability. www.undrr.org/terminology/vulnerability

Zhou, H., Wang, X., \& Wang, J. (2016). A way to sustainability: Perspective of resilience and adaptation to disasters. Sustainability, 8. www.researchgate.net/publication/305826804_A_Way_to_Sustain ability_Perspective_of_Resilience_and_Adaptation_to_Disaster/link/57a368df08ae455e852fe4b0/ download 